\title{
Universos fractales de materia y antimateria y el neutrovacío
}

\section{Fractal Universes of Matter and Antimatter and Neuter Vacuum}

\author{
Enrique Álvarez Vita ${ }^{1}$
}

http://dx.doi.org/10.21503/CienciayDesarrollo.2015.v18i1.06

\section{RESUMEN}

En el presente ensayo proponemos un modelo físico en el que las partículas y antipartículas del vacío cuántico, que a su vez contendrían subniveles de vacío cuántico, polarizarían las cargas eléctricas y de color de leptones y quarks, en una secuencia infinita convergente. En una perspectiva filosófica, el modelo permite sustentar la posible existencia de un universo que sería un vacío neutralizado o neutrovacío, de carácter nouménico, que se manifestaría como dualidad fenoménica en infinitos universos de materia y antimateria de naturaleza fractal, contenidos unos dentro de otros, conformando una sola realidad.

Palabras clave: renormalización, polarización cuántica, universos de materia y antimateria, vacío cuántico fractal, cargas vectoriales, neutrovacio.

\section{ABSTRACT}

In this assay, we offer a physical model in which the particles and antiparticle of vacuum state, which in turn contain sublevels of vacuum state, polarize electric leptons and quark color loads, converging in an infinite sequence. In a philosophical perspective, the model can support the possible existence of a universe that would be neutralized vacuum, noumenal nature that is manifest as phenomenal duality in infinite universes of matter and antimatter of fractal nature, contained within one another, forming a single reality.

Key words: renormalization, quantum polarization, universes of matter and antimatter, fractal quantum vacuum, vector loads, neuter vacuum.

\section{INTRODUCCIÓN}

Tratándose de un ensayo, no es nuestra intención hacer una exposición técnica con todos los aspectos matemáticos del modelo propuesto. Sin embargo, creemos conveniente introducir algunas ecuaciones básicas no tensoriales y gráficas que sustentan nuestra tesis. No obstante, si su comprensión presenta alguna dificultad, bastará con entender los conceptos fundamentales que son fácilmente asimilables.

El trabajo está divido en tres partes, las dos primeras de carácter científico y la última filosófico. La primera trata sobre un nuevo enfoque al problema de la renormalización del electrón o de

1 Miembro de la Sociedad Brasileira Para o Progresso da Ciência (SBPC). Presidente fundador del Centro de Estudios Aeroespaciales y Ciencias del Espacio (CEACE). Miembro del Instituto Peruano de Astronomía (IPA). Miembro de la Asociación Peruana de Historia de la Ciencia (APEDHIC). Vicepresidente de la Academia Peruana de Filosofía y Ciencia (APERFYLC).E-mail:ejualvi@hotmail.com 
cualquier partícula elemental, y el posible origen de las fuerzas fundamentales de la naturaleza. La segunda sobre una breve introducción al álgebra de vectores complejos, reales e imaginarios, y su aplicación a las cargas electrostáticas, nucleares y gravitatorias. La tercera sobre un análisis filosófico de las consecuencias de las dos primeras y la propuesta de un nuevo modelo del universo.

En las últimas décadas, los físicos se han abocado a la ardua tarea de unificar las fuerzas o interacciones fundamentales de la naturaleza: la fuerza de gravedad, la electromagnética, y las fuerzas nucleares débil y fuerte. Con Maxwel se dio el primer paso al unificar la electricidad con el magnetismo en una sola teoría, el electromagnetismo. Posteriormente Glashow, Salam y Weinberg unificaron el electromagnetismo a la fuerza débil, la llamada fuerza electrodébil. La teoría cuántica de campos es una de las más exitosas creadas por el intelecto humano. La concordancia entre los cálculos teóricos y la observación experimental es de una precisión extraordinaria, una parte en $10^{11}$, lo que ha permitido notables aplicaciones en el campo de la tecnología. Para alcanzar este singular éxito, tuvo que superar grandes dificultades a nivel teórico, debido a la presencia de cantidades infinitas que amenazaba con sumergir a la teoría en el caos. En esta breve ponencia, queremos hacer un esbozo de un modelo teórico de carácter científico desarrollado por nosotros, que conduciría a la posibilidad de integrar la gravedad a las demás fuerzas de la naturaleza. Siendo así, expondremos apenas algunos conceptos fundamentales y haremos un enfoque desde una perspectiva filosófica basada en el modelo propuesto, de la naturaleza del universo.

Introduciéndonos en el tema, existen en la naturaleza dos tipos de partículas: los fermiones, de espín fraccionario que conforman la materia, y los bosones, de espín entero, que son los responsables de transmitir las fuerzas fundamentales de la naturaleza. En el grupo de los fermiones, los quarks son partículas elementales que conforman los hadrones, que son partículas compuestas que, al igual que los quarks, reaccionan ante la fuerza fuerte, es decir, aquella fuerza que mantiene unidos a los quarks y al núcleo del átomo, neutralizando la repulsión electrostática de los protones que tienen carga positiva, y por tanto, tienden a separarse. Los hadrones a su vez se presentan en dos variedades: los mesones, constituidos por dos quarks, y los bariones, integrados por tres quarks, como los protones y neutrones que conforman el núcleo atómico. Los quarks junto con los electrones, que son leptones, es decir, partículas que no reaccionan ante la fuerza fuerte, son considerados partículas puntuales sin estructura interna. Los físicos llegaron a esta conclusión debido a la naturaleza repulsiva de la fuerza electrostática que haría despedazar las partículas, lo cual no sucede.

Esta particularidad, sin embargo, crea un serio problema al momento de calcular la energía del campo electrostático de la partícula. Un simple cálculo muestra que la energía disminuye en razón inversamente proporcional a la distancia al centro de la partícula. Como la partícula es puntual, a medida que la distancia se aproxima al centro, la energía se dispara hacia el infinito. Enfrentarse al infinito ha sido una experiencia dura para matemáticos y físicos, y no digamos para filósofos y teólogos. Ahora bien, la teoría especial de la relatividad establece que la masa y la energía son equivalentes, como se expresa en la ecuación $E=m c^{2}$, donde $E$ es la energía, $m$ la masa y $c$ la velocidad de la luz en el vacío. En consecuencia, si la energía se dispara al infinito, debe ocurrir lo mismo con la masa, haciendo que la partícula se torne infinitamente pesada. De hecho, no ocurre así, ya que al medir la masa del electrón se obtienen valores muy pequeños. Algo anda mal en la teoría.

Para superar el problema, los físicos optaron por modificar la escala de medida, ignorando el infinito que surgía en los cálculos y estableciendo 
como nuevo marco de referencia el valor medido experimentalmente. Es lo que se conoce con el nombre de renormalización. A partir de los valores obtenidos experimentalmente, los físicos prosiguieron con sus cálculos, obteniendo resultados con una exactitud asombrosa. Prácticamente todos los avances de la ciencia y tecnología contemporáneas, se debe a la extraordinaria precisión de los cálculos y la concordancia con los experimentos de la mecánica cuántica de campos. Los diagramas de Feynman, notable físico y premio Nobel, constituyen una herramienta importante para el análisis de los procesos cuánticos. No obstante, a nivel conceptual, el problema subsiste. Algunos físicos, como el propio Feynman, que calificó la renormalización como un proceso chiflado, Hawking y Davis, y filósofos como Miró Quesada, entre otros, consideran que la renormalización carece de sólidos fundamentos matemáticos. El delta de Dirac, por ejemplo, que no es propiamente una función matemática, sirvió de base para la renormalización. Dirac inclusive, se refería a ella como el proceso de barrer los infinitos debajo de la alfombra. En la teoría de supercuerdas y la teoría $\mathrm{M}$, la más reciente de las teorías de cuerdas que incluye diez dimensiones espaciales y una temporal, se considera al electrón y demás partículas como cuerdas vibrantes a escalas de longitud de Planck, magnitud extremadamente pequeña, y no como una partícula puntual según el modelo estándar de la física, no obstante dentro de la comunidad científica esta teoría es considerada especulativa por las dificultades técnicas de su comprobación experimental. En la gravedad cuántica de bucles el espaciotiempo está cuantizado y se fija un límite mínimo para el espacio y el tiempo, que serían la distancia y el tiempo de Planck, se trata de un modelo sobre la gravedad cuántica y no de unificación de las fuerzas fundamentales de la naturaleza.

A fin de proporcionar a la teoría cuántica un fundamento conceptual consistente, considerando al electrón como partícula puntual sin estructura interna, elaboramos un modelo matemático basado en las propiedades del vacío cuántico, que serían la clave de la solución al problema. El aporte original de la tesis propuesta, es postular la existencia de subniveles de energía del vacío cuántico, lo cual permitiría incorporar la gravedad a las demás fuerzas fundamentales de la naturaleza, lo que tendría implicancias no sólo científicas sino filosóficas, como veremos más adelante. El electrón atrae positrones virtuales y repele electrones virtuales, lo que atenúa su carga eléctrica, fenómeno conocido como polarización cuántica o apantallamiento del electrón. Según nuestro modelo, esta capa de positrones virtuales atrae electrones virtuales y repele positrones virtuales de un segundo nivel de energía del vacío cuántico, aumentando la carga eléctrica del electrón, de acuerdo a una determinada ley en función de la distancia. Este proceso continuaría indefinidamente en una serie matemática infinita convergente de naturaleza fractal, que produciría una polarización del campo electrogravitatorio a distancias muy cortas, neutralizando el campo a una distancia nula del electrón. La energía de cada nivel se calcula por la integral definida entre dos puntos de la energía del nivel anterior, siendo la energía neta la sumatoria de estas integrales. Nuestros cálculos nos conducen de manera natural a valores finitos para la energía. Cuando $r \rightarrow 0$, la energía potencial del electrón se convierte en su energía intrínseca $E=m_{e} c^{2}$, siendo $m_{e}$ la masa del electrón, que es precisamente la ecuación de Einstein de la energía del electrón, es decir, cuando su energía cinética autoinducida es nula, en concordancia con la teoría y la observación experimental.

Consideremos en primer lugar, los campos gravitatorio y electrostático del electrón. Según la física clásica, las fuerzas de atracción gravitatoria $\boldsymbol{F}_{g_{r}}^{e^{-}}$y repulsión eléctrica $\boldsymbol{F}_{e_{r}}^{e^{-}}$que el electrón ejerce sobre sí mismo, considerado como un cascarón esférico de radio $r$, está dada por: 


$$
\begin{gathered}
\boldsymbol{F}_{g_{r}}^{e^{-}}=-\frac{G m_{e}^{2}}{r^{2}} \boldsymbol{r} \\
\boldsymbol{F}_{e_{r}}^{e^{-}}=\frac{k e^{-^{2}}}{r^{2}} \boldsymbol{r}
\end{gathered}
$$

siendo $G$ la constante de gravitación universal, me la masa del electrón, $k$ la constante electrostá- tica coulombiana $e^{-}$, la carga del electrón y $r$ un vector unitario que indica el sentido de la fuerza, positiva si es repulsiva y negativa si es atractiva. Los caracteres en negrita representan magnitudes vectoriales. La fuerza resultante $\boldsymbol{F}_{r}^{e^{-}}$entre ambas fuerzas está dada por:

$$
\boldsymbol{F}_{r}^{e^{-}}=\frac{k e^{-^{2}}}{r^{2}} \boldsymbol{r}-\frac{G m_{e}^{2}}{r^{2}} \boldsymbol{r}
$$

La energía potencial resultante $U_{p_{a b 0}}^{e^{-}}$entre las distancias a y $b$ del electrón está dada por:

$$
U_{p_{a b 0}}^{e^{-}}=\int_{a}^{b}\left(\frac{k e^{-2}}{r^{2}}-\frac{G m_{e}^{2}}{r^{2}}\right) d r=\left(k e^{-^{2}}-G m_{e}^{2}\right)\left(\frac{1}{a}-\frac{1}{b}\right)
$$

Multiplicando y dividiendo por $\varphi$, una constante de longitud vinculada al electrón, por ahora indeterminada, tenemos:

$$
\begin{gathered}
U_{p_{a b 0}}^{e^{-}}=\frac{k e^{-2}-G m_{e}^{2}}{\varphi}\left(\frac{\varphi}{a}-\frac{\varphi}{b}\right)=\frac{k e^{-2}-G m_{e}^{2}}{\varphi}\left(\varnothing_{a}-\emptyset_{b}\right) \\
=\int_{\emptyset_{a}}^{\emptyset_{b}} \frac{k e^{-^{2}}-G m_{e}^{2}}{\varphi} d \emptyset
\end{gathered}
$$

siendo $\emptyset_{r}=\varphi / r$. Este cambio de variable es esencial en el desarrollo del modelo propuesto. La expresión $\left(k e^{-2}-G m_{e}^{2}\right) / \varphi$ es una energía potencial elctrogravitatoria asociado al electrón, invariante respecto a la distancia $r$. La integral respecto a la función $\emptyset$ en la ecuación, define la energía potencial electrogravitatoria entre dos puntos situados a las distancias $a$ y $b$ de la partícula.

Se ha comprobado experimentalmente mediante el acelerador de partículas japonés TRISTAN que el electrón atrae positrones virtuales y repele electrones virtuales presentes en el vacío cuántico, que atenúan la intensidad del campo electrostáti- co, produciendo un efecto de apantallamiento o polarización cuántica. Lo contrario sucede con la fuerza gravitatoria, que como consecuencia de la repulsión entre materia y antimateria, efecto que más adelante veremos, atrae electrones virtuales y repele positrones virtuales, lo que refuerza la intensidad del campo gravitatorio. No obstante, siendo la fuerza electrostática mucho más intensa que la gravitatoria, los positrones virtuales serán atraídos y los electrones virtuales repelidos, produciéndose un apantallamiento electrogravitatorio. Cada positrón virtual de este nuevo campo, que denominamos campo de primer nivel del vacío cuántico, generará una energía potencial $U_{p_{a b 1}}^{e^{-}}$que disminuirá la energía $U_{p_{a b 0}}^{e^{-}}$. 
Ahora bien, hemos visto que la energía potencial $U_{p_{a b 0}}^{e^{-}}$se obtuvo por integración de la energía potencial $\left(k e^{-2}-G m_{e}{ }^{2}\right) / \varphi$ respecto a $\emptyset$. Análogamente, la energía potencial $U_{p_{a b 1}}^{e^{-}}$generada por los positrones virtuales deberá obtenerse por integración de la energía potencial $U_{p_{a b 0}}^{e^{-}}$respecto a $\emptyset$. En consecuencia, la energía potencial $U_{p_{a b 1}}^{e^{-}}$ de este nuevo campo estaría dada por:

$$
U_{p_{a b 1}}^{e^{-}}=\int_{\emptyset_{a}}^{\emptyset_{b}}-\frac{\left(k e^{-^{2}}-G m_{e}{ }^{2}\right) \emptyset}{\varphi} d \emptyset=-\frac{k e^{+^{2}}-G m_{e}{ }^{2}}{2 ! \varphi}\left(\emptyset_{a}{ }^{2}-\emptyset_{b}{ }^{2}\right)
$$

siendo $e^{+}$la carga del positrón y el signo de $U_{p_{a b 1}}^{e^{-}}$negativo. De modo análogo, estos positrones virtuales atraerán electrones virtuales y repelerán positrones virtuales de un campo de segundo nivel, que aumentará la intensidad del campo electrogravitatorio del electrón, de manera que este nuevo campo generará una energía potencial $U_{p_{a b 2}}^{e^{-}}$que incrementará la energía potencial $U_{p_{a b 0}}^{e^{-}}$del electrón, que análogamente estará dada por la integración de $U_{p_{a b 1}}^{e^{-}}$respecto a $\emptyset$. Consecuentemente:

$$
U_{p_{a b 2}}^{e^{-}}=\int_{\emptyset_{a}}^{\emptyset_{b}} \frac{\left(k e^{+^{2}}-G m_{e}^{2}\right) \emptyset^{2}}{2 ! \varphi} d \emptyset=\frac{k e^{-^{2}}-G m_{e}{ }^{2}}{3 ! \varphi}\left(\varnothing_{a}{ }^{3}-\emptyset_{b}{ }^{3}\right)
$$

Este proceso continuará indefinidamente. En general, una energía potencial de enésimo nivel $U_{p_{a b n}}^{e^{-}}$ estará dada por:

$$
\begin{aligned}
& U_{p_{a b n}}^{e^{-}}=\int_{\emptyset_{a}}^{\emptyset_{b}}(-1)^{n} \frac{\left(k e^{ \pm^{2}}-G m_{e}^{2}\right) \emptyset^{n}}{n ! \varphi} d \emptyset \\
& =(-1)^{n} \frac{k e^{ \pm^{2}}-G m_{e}^{2}}{(n+1) ! \varphi}\left(\emptyset_{a}^{n+1}-\emptyset_{b}{ }^{n+1}\right)
\end{aligned}
$$

Es decir, la energía potencial de nivel $n$ entre los puntos $a$ y $b$ se obtiene por la integral definida entre dichos puntos de la energía potencial de nivel $n-1$.

La energía potencial neta $U_{p_{a b}}^{e^{-}}$estará dada por la sumatoria de todas las energías potenciales de enésimo nivel hasta el infinito, es decir:

$$
\begin{gathered}
U_{p_{a b}}^{e^{-}}=\sum_{n=0}^{\infty}(-1)^{n} \frac{k e^{ \pm^{2}}-G m_{e}^{2}}{(n+1) ! \varphi}\left(\emptyset_{a}{ }^{n+1}-\emptyset_{b}{ }^{n+1}\right) \\
=\frac{k e^{-^{2}}-G m_{e}^{2}}{\varphi}\left(e^{-\emptyset_{b}}-e^{-\emptyset_{a}}\right) \\
=\frac{k e^{-2}-G m_{e}^{2}}{\varphi}\left(e^{-\frac{\varphi}{b}}-e^{-\frac{\varphi}{a}}\right)
\end{gathered}
$$


siendo $e$ la base de los logaritmos neperianos y considerando que la carga $e^{-}$del electrón y $e^{+}$ positrón son de la misma magnitud y podemos representarlas como $e^{-}$.

Ahora bien, la ecuación nos da la diferencia de energía potencial electrogravitatoria entre dos puntos situados a distancias $a$ y $b$ del electrón. Podemos asignar un valor al potencial en un punto a una distancia $r$ de la partícula, para lo cual es necesario elegir otro punto de referencia arbitrario al que se le asigna el llamado potencial cero. Para satisfacer esta condición, dicho punto debe hallarse a una distancia infinita. Si asignamos el punto $r$ en $a$ y el infinito en $b$ obtendremos el potencial $U_{p_{r \infty}}^{e^{-}}$o simplemente $U_{p_{r}}^{e^{-}}$, que nos dará:

$$
U_{p_{r}}^{e^{-}}=\frac{k e^{-^{2}}-G m_{e}^{2}}{\varphi}\left(1-e^{-\frac{\varphi}{r}}\right)
$$

O bien:

$U_{p_{r}}^{e^{-}}=\left(k e^{-^{2}}-G m_{e}{ }^{2}\right)\left(\frac{1}{r}-\frac{\varphi}{2 ! r^{2}}+\frac{\varphi^{2}}{3 ! r^{3}}-\cdots\right)$

Cuando $r$ es mucho mayor que $\varphi$, entonces los términos de la sucesión dentro del paréntesis a partir del segundo término pueden despreciarse, con lo cual tendremos:

$$
U_{p_{r}}^{e^{-}}=\frac{k e^{-^{2}}}{r}-\frac{G m_{e}^{2}}{r}
$$

resultado que coincide con el de la física clásica para distancias relativamente grandes, como caso particular. Si en la ecuación de la energía potencial $r \rightarrow 0$, tendremos:

$$
U_{p_{0}}^{e^{-}}=\frac{k e^{-^{2}}-G m_{e}^{2}}{\varphi}
$$

Este resultado nos conduce a un valor finito para el potencial a una distancia nula del electrón, a diferencia de la física clásica que arroja un va- lor infinito. La energía potencial $U_{p_{0}}^{e^{-}}$es precisamente la misma que genera por integraciones sucesivas la energía potencial electrogravitatoria neta del electrón y de los positrones y electrones virtuales, invariante respecto a la distancia $r$. La figura muestra la gráfica sin escala de la energía potencial $U_{p_{r}}^{e^{-}}$.

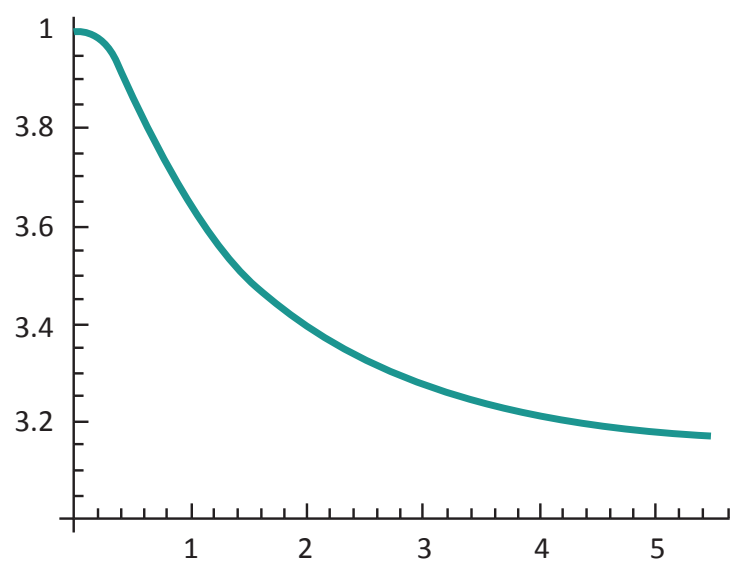

Ahora bien, como mencionamos anteriormente, en mecánica relativista masa y energía son equivalentes. Eso implica que cualquier sistema físico con energía debería presentar una cierta inercia, de modo que al tratar de mover el electrón éste arrastraría a su campo electrogravitatorio generando así una inercia, que sería vista como una masa efectiva. En consecuencia, la energía del campo electrogravitatorio $U_{p_{0}}^{e^{-}}$de un electrón medida por un observador en reposo es equivalente a su energía intrínseca $E=m_{e} c^{2}$. Reemplazando este valor en la ecuación de la energía tendremos:

$$
m_{e} c^{2}=\frac{k e^{-^{2}}-G m_{e}^{2}}{\varphi}
$$

de donde:

$$
\varphi=\frac{k e^{-^{2}}-G m_{e}^{2}}{m_{e} c^{2}}=\frac{\left[\alpha-\alpha_{g}\right] \hbar}{m_{e} c}
$$

siendo $\alpha=k e^{-2} / \hbar c$ la constante de estructura fina electromagnética del electrón, de gran 
importancia en cosmología, $\alpha_{g}=G m_{e}^{2} / \hbar c$ la constante de acoplamiento gravitacional del electrón y $\hbar$ la constante reducida de Planck.

Derivando la ecuación de la energía con respecto a $r$, obtenemos la fuerza electrogravitatoria $\boldsymbol{F}_{r}^{e^{-}}$ autoinducida, es decir, sobre sí misma, del electrón a una distancia $r$, es decir, la fuerza electrostática repulsiva de signo positivo más la fuerza gravitatoria atractiva de signo negativo:

$$
\begin{aligned}
& \boldsymbol{F}_{r}^{e^{-}}=\frac{k e^{-^{2}}-G m_{e}{ }^{2}}{r^{2}} e^{-\frac{\varphi}{r}} \boldsymbol{r} \\
& =\frac{k e^{-{ }^{2}}-G m_{e}{ }^{2}}{r^{2}} e^{-\frac{[\alpha-\alpha g] \hbar}{m_{e} c r}} \boldsymbol{r}
\end{aligned}
$$

Este resultado es consecuente con el hecho de que el electrón no estalle bajo los efectos de su propia carga eléctrica repulsiva, al neutralizarse la intensidad del campo electrogravitatorio a una distancia nula de la partícula. Cabe señalar que para superar este inconveniente, los físicos propusieron que el electrón es una partícula puntual sin estructura interna. Pero esta hipótesis implica que la energía potencial electrogravitatoria se torna infinita. Esta relación es fundamental porque la carga eléctrica del electrón atrae antipartículas o positrones virtuales del vacío cuántico que polarizan no solo la carga eléctrica sino también la gravedad, como veremos más adelante.

Desarrollando en serie la ecuación tenemos:

$\boldsymbol{F}_{r}^{e^{-}}=\frac{k e^{-^{2}}-G m_{e}^{2}}{r^{2}}\left(1-\frac{\varphi}{r}+\frac{\varphi^{2}}{2 ! r^{2}}-\cdots\right) \boldsymbol{r}$

Si $r$ es mucho mayor que $\varphi$, podemos despreciar los términos dentro del paréntesis a partir del segundo término, obteniéndose el resultado clásico de la ecuación de la fuerza electrogravitatoria, como caso particular. Cuando $r \rightarrow 0$, la fuerza
$\boldsymbol{F}_{r}^{e^{-}}$se anula, a diferencia del modelo clásico que da un resultado infinito. La figura representa la gráfica sin escala de la ecuación según el modelo propuesto.

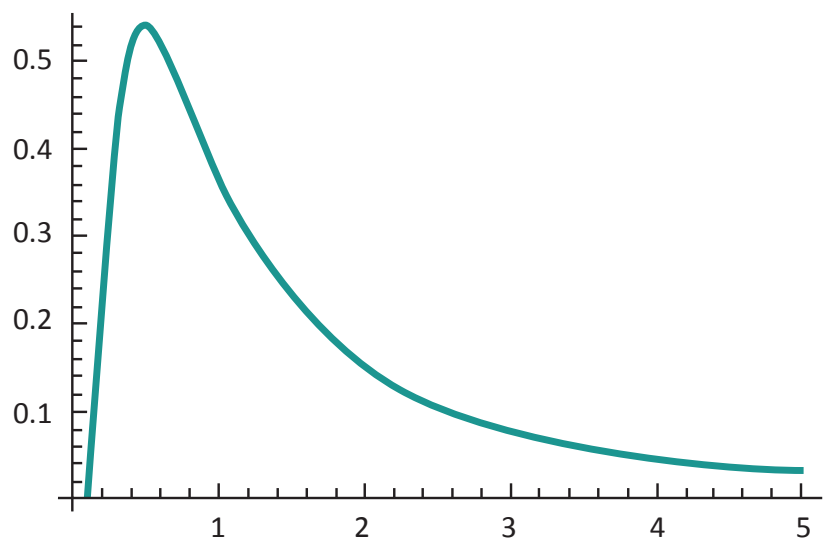

Según el modelo estándar de la física de partículas, el neutrino fue concebido inicialmente como un fermión sin masa ni carga eléctrica. Experimentos posteriores demostraron que el neutrino poseería una masa muy pequeña. En el modelo propuesto, debería poseer también una pequeñísima carga eléctrica, la suficiente para polarizar su gravedad, es decir, $k q_{v_{e}}{ }^{2}>G m_{v_{e}}{ }^{2}$, siendo $q_{v_{e}} \mathrm{y}$ $m_{v_{e}}$ la carga y la masa del neutrino respectivamente, de donde $q_{v_{e}}>m_{v_{e}} \sqrt{G / k}$, es decir, $q_{v_{e}}>2,46$ $\mathrm{x} 10^{-28} e^{-}$, cantidad que perderían las partículas beta en la desintegración del neutrón sin violar el principio de conservación de la carga. Con velocidades cercanas a la luz y la disminución del campo eléctrico a cortas distancias debido al factor exponencial, los neutrinos son prácticamente insensibles a la acción de la fuerza electrostática y en consecuencia casi no interactúan con la materia. El efecto acumulado de las fuerzas repulsivas entre las cargas de los neutrinos podría ser el origen de la energía oscura y la expansión acelerada del universo, teniendo en cuenta la alta densidad de los neutrinos en la fase inicial de la expansión y el incremento y posterior disminución de estas fuerzas con la distancia, como muestra la figura. Podría tratarse también de la carga electrodébil que poseen los neutrinos. De acuerdo al modelo 
propuesto, la ausencia de una carga fractal convergente superior a la fuerza gravitatoria en el neutrino implicaría una energía o masa infinitas.

Ahora bien, si consideramos que la constante de estructura fina del electrón $\alpha$ es $10^{43}$ mayor que la constante de acoplamiento gravitacional del electrón $\alpha_{g}$, podemos despreciar este término en las ecuaciones sin perder precisión, de manera que la intensidad del campo electrostático $E_{r}^{e^{-}}$ del electrón en unidades gausianas, despreciando el campo gravitatorio extremadamente débil, estaría dada por:

$$
\boldsymbol{E}_{r}^{e^{-}}=\frac{e^{-}}{4 \pi \varepsilon_{0} r^{2}} e^{-\frac{\alpha \hbar}{m_{e} c r}} \boldsymbol{r}
$$

que puede referirse también a una carga cualquiera $q$, siendo $\varepsilon_{0}$ la constante de permitividad en el vacío. La expresión $\alpha \hbar / m_{e} c$ corresponde al radio clásico del electrón $r_{e}$. A grandes distancias el exponencial tiende a la unidad y la ecuación se aproxima a la fórmula de la intensidad del campo electrostático de la física clásica.

\section{La belleza como guía de la ciencia}

Quisiera hacer una breve reflexión con relación a esta ecuación. Esta fórmula fue publicada por primera vez en nuestro ensayo "La belleza como guía de la ciencia”, en la décimo primera edición de la revista Tradición editada por la Universidad Ricardo Palma, en el que sustentamos que muchos de los descubrimientos científicos han sido inspirados en un sentido de lo estético. Permítanme exponer algunos de los conceptos contenidos en este ensayo. La belleza y simetría ocultas en las matemáticas, han servido de guía para el investigador, algunas veces en aparente contradicción con los mismos experimentos y el sentido común. Probablemente para muchos les parecerá extraño que el criterio de belleza haya contribuido al desarrollo de la ciencia. Pues la verdad es que muchos científicos a través de la historia han elaborado sus teorías guiados por un sentido de la estética. Uno de los ejemplos más notorios es el de Einstein, creador de la teoría de la relatividad. Inspirado en un concepto de simetría y belleza, Einstein seleccionaba entre una variedad de soluciones a sus ecuaciones de campo, aquellas que a su criterio le parecían más estéticas. Indudablemente la simetría juega un rol fundamental. A medida que la ciencia ha ido avanzando en su conocimiento de la naturaleza, ha descubierto simetrías ocultas en las leyes que la gobiernan. Hay una profunda conexión entre la simetría geométrica y las leyes de conservación de la física. En un sistema aislado, la energía, la cantidad de movimiento y el momento angular permanecen invariables en el tiempo. Las leyes de conservación derivan de las leyes de Newton, y su posterior reformulación por los matemáticos Lagrange y Hamilton, revelaron la presencia de esa simetría.

Esta simetría se nos revela precisamente a través del lenguaje matemático. Las matemáticas poseen en sí mismas una belleza que muchas veces permanece oculta a los ojos del profano. En sus extraños y sofisticados símbolos, se esconde la belleza que nos inspira una melodía, una pintura, una poesía, una escultura o una obra arquitectónica. Y en ese íntimo vínculo con la naturaleza, se nos manifiesta en toda su grandeza. El gran matemático Poincaré, el astrofísico Paul Davis y el filósofo Francisco Miró Quesada, entre otros, han expresado el mismo concepto. Para algunos filósofos, la belleza sólo existe en función del objeto. En este caso, el objeto es una ecuación matemática. En el proceso de creación de una teoría científica, las ecuaciones surgen como consecuencia de este proceso, y la belleza que captamos en ellas, nos induce a concluir que nos encontramos en la dirección correcta. Al término de esta elaboración, obtenemos una ecuación final cuya contemplación nos produce el mismo efecto que la culminación de una obra de arte. En este sentido decimos que la belleza es una guía para la ciencia. 
El notable matemático y premio Nobel de física, Paul Dirac, llegó a afirmar que la belleza de una teoría científica es más importante aún que la prueba experimental. Dirac tenía sus motivos: cuando desarrolló su ecuación de onda para el electrón, incorporando la teoría de la relatividad, descubrió que debía existir una partícula semejante al electrón pero con carga eléctrica positiva, es decir un antielectrón o positrón como se le bautizó posteriormente. Su ecuación de onda poseía una simetría más poderosa que la ecuación de onda de Schrödinger y fue calificada como una verdadera obra de arte. No obstante, algunos científicos la acogieron con escepticismo. Heisenberg, premio Nobel de física y descubridor del famoso principio de incertidumbre, llegó a afirmar que Dirac había escrito el capítulo más triste de la física moderna. A pesar de ello y la presión de algunos colegas, Dirac no se amilanó. Sin embargo, hubo que esperar algunos años hasta que Anderson, un físico experimental, demostrara en su laboratorio la existencia del positrón. Dirac tenía razón. Ambos científicos, uno teórico y otro experimental, ganaron el premio Nobel por ese descubrimiento.

Para el positivista Mario Bunge, sin embargo, la belleza de una teoría no determina la validez de la misma, sino la prueba experimental, la cual puede ser siempre refutada, criterio acorde con la metodología científica y el falsacionismo de Popper y Lakatos. Para Bunge la intuición no es un método válido para el conocimiento científico. No obstante, las experiencias mencionadas demuestran que el sentido de lo estético ha jugado un rol importante en la elaboración de algunas teorías científicas que posteriormente fueron confirmadas experimentalmente. Cabe señalar que nosotros intuimos que la ecuación en referencia debería contener estos elementos dispuestos de manera estética y elegante, sin conocer su estructura definitiva. Luego de varias tentativas llegamos por fin a este resultado, que al contemplarlo nos produce una sensación de belleza, lo cual nos induce a pensar que la ecuación es correcta. Hace cerca de dos décadas escribimos parte de este ensayo para un cenáculo de filosofía. En aquella época no podíamos imaginarnos que varios años después, hace apenas algunos meses, obtendríamos esta ecuación. Lo que fue una descripción de la experiencia estética de algunos científicos al desarrollar sus teorías, se convertiría con el tiempo en una premonitoria y modesta vivencia personal. Si estamos equivocados o no, el tiempo lo dirá.

\section{La energía no se conserva}

La energía potencial electrogravitatoria $U_{p_{r}}^{e^{-}}$autoinducida del electrón a una distancia $r$ estaría dada por:

$$
U_{p_{r}}^{e^{-}}=m_{e} c^{2}\left(1-e^{-\frac{[\alpha-\alpha g] \hbar}{m_{e} c r}}\right)
$$

Cuando $r \rightarrow 0$, la energía potencial del electrón se convierte en su energía intrínseca $E=m_{e} c^{2}$, que es precisamente la ecuación de Einstein de la energía del electrón, es decir, cuando su energía cinética autoinducida es nula, en concordancia con la teoría y la observación experimental. El modelo propuesto implica que los positrones virtuales en cada nivel producen asimismo fotones virtuales que generan energías negativas que polarizan la energía originada por los fotones virtuales del electrón, evitando la energía infinita como consecuencia de la autoinducción de estos fotones, proceso que ocurriría con todos los fermiones y sus respectivas antipartículas y bosones virtuales.

El laplaciano $\nabla^{2}$ o divergencia del gradiente de la energía potencial electrogravitatoria autoinducida del electrón a una distancia $r$ está dado por: 


$$
\begin{aligned}
\nabla^{2} U_{p_{r}}^{e^{-}} & =\left(\frac{\partial^{2}}{\partial x^{2}}+\frac{\partial^{2}}{\partial y^{2}}+\frac{\partial^{2}}{\partial z^{2}}\right)\left[m_{e} c^{2}\left(1-e^{-\frac{[\alpha-\alpha g] \hbar}{m_{e} c r}}\right)\right] \\
& =-\frac{\left(k e^{-2}-G m_{e}^{2}\right)^{2}}{m_{e} c^{2} r^{4}} e^{-\frac{[\alpha-\alpha g] \hbar}{m_{e} c r}}
\end{aligned}
$$

El significado físico de este resultado de signo negativo es que existe un sumidero en el fluido electrogravitatorio, más intenso en las proximidades del electrón, como consecuencia de la polarización cuántica, donde la energía negativa de las antipartículas virtuales apantallan la energía positiva electrogravitatoria. En los modelos clásico y relativista, la divergencia es nula y en consecuencia no existe ni fuente ni sumidero, lo que conduce a valores infinitos en el modelo clásico y a una singularidad espaciotemporal en el modelo relativista, al no considerar los efectos de la polarización cuántica.

El principio de polarización cuántica sería válido para cualquier fuerza fundamental de la naturaleza atractiva o repulsiva. En el modelo clásico del átomo de Bohr, por ejemplo, su primer postulado se modificaría de la siguiente manera:

$$
\frac{k Z e^{-^{2}}}{r^{2}} e^{-\frac{k e^{-2}}{m_{e} c^{2} r}}=\frac{1}{2} m_{e} v^{2}
$$

donde $Z$ es el número atómico y $v$ la velocidad del electrón, modificándose también sus siguientes postulados. El factor exponencial del modelo propuesto afectaría igualmente las ecuaciones de onda de Schorödinger, Pauli y Dirac, que involucran fuerzas centrales.

Los positrones virtuales serían partículas cuyo tiempo transcurre del pasado hacia el futuro, pero en sentido contrario al flujo del tiempo de los electrones, conservando la simetría CPT: carga, paridad o imagen especular invertida y tiem- po. De manera que de acuerdo a nuestro modelo, las antipartículas virtuales no solo polarizan la fuerza gravitatoria sino también la curvatura espaciotemporal en las ecuaciones de campo de Einstein, contrarrestando el retardo temporal por efecto de la gravedad. La polarización cuántica afectaría no solo al electrón y demás leptones, sino también a los quarks y la fuerza nuclear, así como la gravedad en general, según nuestros cálculos, representando un avance hacia la unificación de las fuerzas fundamentales de la naturaleza, uno de los grandes retos de la física en la actualidad. La ecuación de campo de Einstein, confirmada por los experimentos realizados hasta el día de hoy, con una precisión extraordinaria de una parte en $10^{14}$, superior incluso a la de la mecánica cuántica de campos en sus respectivos dominios, pierde validez a distancias extremadamente pequeñas, a escalas de Planck. El principio de conservación de la energía, prácticamente válido a grandes distancias, no se aplica a estas escalas, motivo por el que no se ha logrado hasta el momento una teoría cuántica de la gravedad, que se polariza por la acción de las antipartículas virtuales.

En el caso de los quarks, las cargas de color atraen sus respectivas partículas virtuales anticolor, polarizando también la gravedad. La fuerza fuerte se cancela a nivel del núcleo atómico y las electromagnéticas a nivel atómico y molecular por la neutralización de las cargas de los protones y electrones, con excepción de ciertos materiales y cuerpos masivos con campos electromagnéticos débiles. A diferencia de las otras fuerzas, las tenues fuerzas gravitatorias no se cancelan entre 
sí y su efecto acumulativo las convierten en la fuerza dominante a nivel del cosmos. Nuestros cálculos demuestran que el apantallamiento gravitatorio se puede generalizar a cualquier masa, la gravedad se polariza bajo los efectos cuánticos fractales de las antipartículas virtuales, el infinito queda atrapado y la gravedad capturada.

Según la relatividad general, la gravedad es un efecto de la curvatura espaciotemporal generada por la masa y la energía de las antipartículas virtuales producirían una curvatura espaciotemporal negativa que contrarrestaría la curvatura espaciotemporal positiva, generando un apantallamiento gravitatorio, de modo que la ecuación de campo de Einstein para la curvatura espaciotemporal $G_{u v}$ se modificaría.

Einstein asumió, por el principio de conservación de la energía, que la divergencia de $G_{u v}$, al igual que el laplaciano del potencial gravitatorio de Newton, debería ser nula, es decir, $G_{; v}^{u v}=0$, lo que conlleva, al no existir ni fuente ni sumidero en el flujo gravitatorio, a una singularidad espaciotemporal cuando $r \rightarrow 0$ como mencionamos anteriormente, en el que la curvatura y la atracción gravitatorias se hacen infinitas. En la ecuación tensorial modificada propuesta por nosotros, $G_{; v}^{u v} \neq 0$. El análisis de esta modificación escapa al presente ensayo y será objeto de una próxima publicación.

\section{Cargas vectoriales}

Proponemos asimismo, que las cargas electrostáticas, cromodinámicas y gravitatorias, pueden representarse por medio de vectores complejos cuyo producto escalar determina la atracción, repulsión o inexistencia de ambas. En nuestro trabajo "Introducción al análisis de vectores y tensores complejos" ofrecemos una exposición más detallada sobre el tema, que incluye derivadas e integrales de vectores complejos, operaciones de gradiente, divergencia y rotacional, derivadas covariantes y contravariantes de tensores complejos, etc. Básicamente, todas las operaciones del análisis de vectores y tensores reales son válidas para los vectores y tensores complejos, teniendo en cuenta que estos últimos son el producto de vectores y tensores reales por un escalar complejo. Los vectores complejos definen un campo vectorial de $\mathrm{n}$ dimensiones. En el presente ensayo expondremos algunas nociones básicas para entender el comportamiento de las cargas de las fuerzas fundamentales de la naturaleza. Sean $\boldsymbol{A} e^{i \alpha}=\boldsymbol{A}(\cos \alpha+i \operatorname{sen} \alpha)$ y $\boldsymbol{B} e^{i \beta}=\boldsymbol{B}(\cos \beta+i \operatorname{sen} \beta)$ dos vectores complejos expresados en forma polar, siendo la unidad imaginaria $\mathrm{i}=\sqrt{-1}$ y $e$ la base de los logaritmos neperianos, cuya representación gráfica es la siguiente:

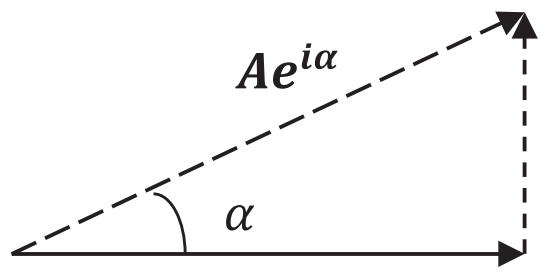

$\boldsymbol{A} \cos \alpha$
$A i \operatorname{sen} \alpha$

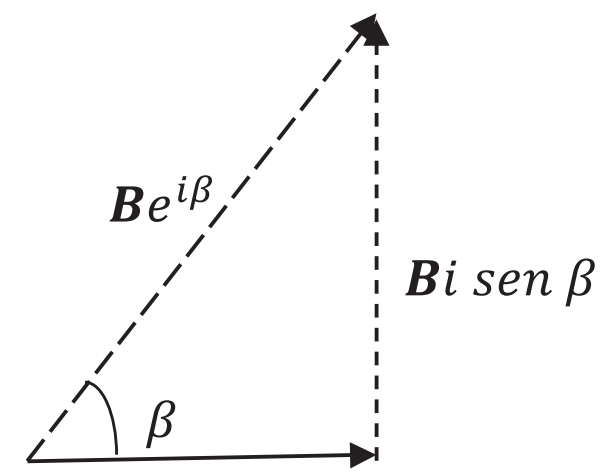

$\boldsymbol{B} \cos \beta$ 
Suponiendo que dos vectores $\boldsymbol{A} e^{i \alpha}$ y $\boldsymbol{B} e^{i \beta}$ formen un ángulo $\theta$, la suma de ambos vectores estará dada por:

$$
\begin{gathered}
\boldsymbol{A} e^{i \alpha}+\boldsymbol{B} e^{i \beta}=\boldsymbol{A}(\cos \alpha+i \operatorname{sen} \alpha)+\boldsymbol{B}(\cos \beta+i \operatorname{sen} \beta) \\
=(\boldsymbol{A} \cos \alpha+\boldsymbol{B} \cos \beta)+i(\boldsymbol{A} \operatorname{sen} \alpha+\boldsymbol{B} \operatorname{sen} \beta)
\end{gathered}
$$

cuya representación gráfica es:

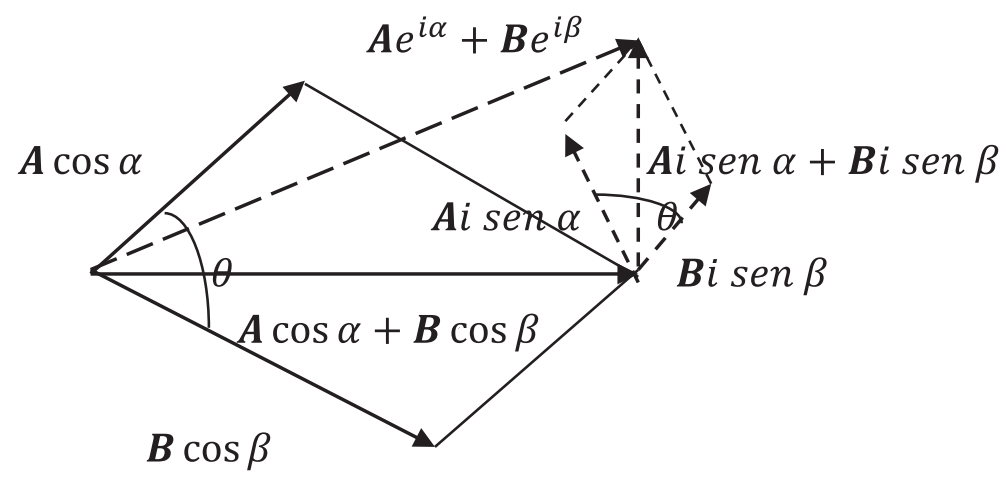

Es decir, la suma de dos vectores complejos es la suma vectorial de sus componentes reales por un lado y la suma vectorial de sus componentes imaginarias por otro, de manera que estas resultantes constituyen las componentes real e imaginaria de un nuevo vector complejo que es la resultante de esta suma, cuyo argumento es el siguiente:

$$
\left|\boldsymbol{A} e^{i \alpha}+\boldsymbol{B} e^{i \beta}\right|=\sqrt{A^{2}+2 A B \cos (\alpha-\beta) \cos \theta+B^{2}}
$$

Cuando $\alpha=\beta$, el argumento es el mismo que el de dos vectores reales.

Su producto escalar está dado por:

$$
\boldsymbol{A} e^{i \alpha} \cdot \boldsymbol{B} e^{i \beta}=A B e^{i(\alpha+\beta)} \cos \theta
$$

Siendo $A$ y $B$ sus respectivos módulos. Su producto vectorial está dado por:

$$
\boldsymbol{A} e^{i \propto} \times \boldsymbol{B} e^{i \beta}=A B e^{i(\alpha+\beta)} \operatorname{sen} \theta \boldsymbol{u}
$$

donde $\boldsymbol{u}$ es un vector unitario perpendicular al plano conformado por ambos vectores, cuyo sentido, al igual que el producto de vectores reales, está determinado por la regla de la mano derecha. Al igual que los vectores reales, los vectores imaginarios conforman un espacio euclidiano n-dimensional, en el que cada vector direccional imaginario conforma con su respectivo vector direccional real un plano vectorial complejo. Los vectores complejos están definidos por la suma de sus correspondientes vectores reales e imaginarios. Los vectores complejos determinan un espacio vectorial complejo. Lo mismo ocurre con los escalares complejos y sus respectivas coordenadas reales e imaginarias.

Ahora bien, podemos representar las cargas eléctricas por medio de dos vectores reales unitarios, $\boldsymbol{A}=\boldsymbol{B}=\boldsymbol{u}, e^{i \alpha} \boldsymbol{u}$ y $e^{i \beta} \boldsymbol{u}$, que forman un ángulo $\theta=0^{\circ}$ o $\theta=180^{\circ}$. Si en el producto escalar $\alpha=\beta=\pi$ y $\theta=0^{\circ}$, tendremos: 


$$
\boldsymbol{u} \cdot \boldsymbol{u}=e^{2 i \pi} \cos 0^{\circ}=+1
$$

es decir, las cargas se repelen. Si $\theta=180^{\circ}$ el producto escalar estará dado por:

$$
\boldsymbol{u} \cdot \boldsymbol{u}=e^{2 i \pi} \cos 180^{\circ}=-1
$$

y las cargas se atraen, como se ilustra en la figura. Este es el comportamiento de las cargas electrostáticas y de las cargas de color con sus respectivos anticolores.

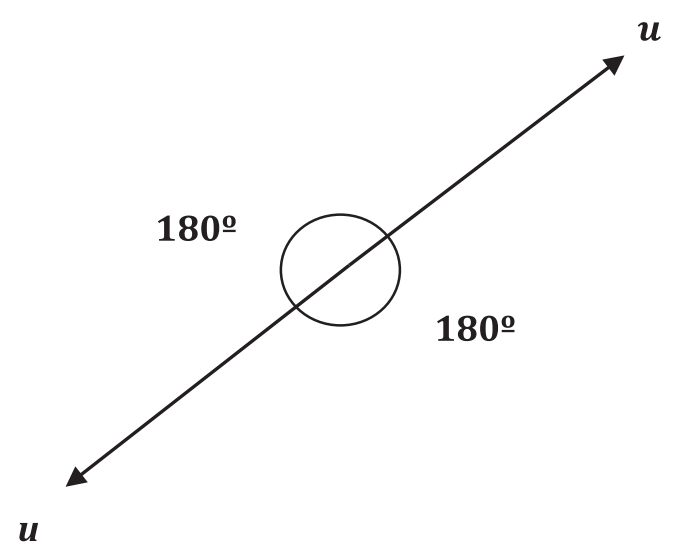

Los bariones poseen tres quarks cuyas cargas están representadas por los colores rojo, azul y verde (o rojo, azul y amarillo), cuya suma da blanco. Estas cargas cambian constantemente de color.Del mismo modo podemos representar las cargas de color por medio de tres vectores reales unitarios, $\boldsymbol{A}=\boldsymbol{B}=\boldsymbol{C}=\boldsymbol{u}, e^{i \alpha} \boldsymbol{u}, e^{i \beta} \boldsymbol{u}$ y $e^{i \gamma} \boldsymbol{u}$, que forman entre sí un ángulo de donde $\alpha=\beta=\gamma=\pi$ :

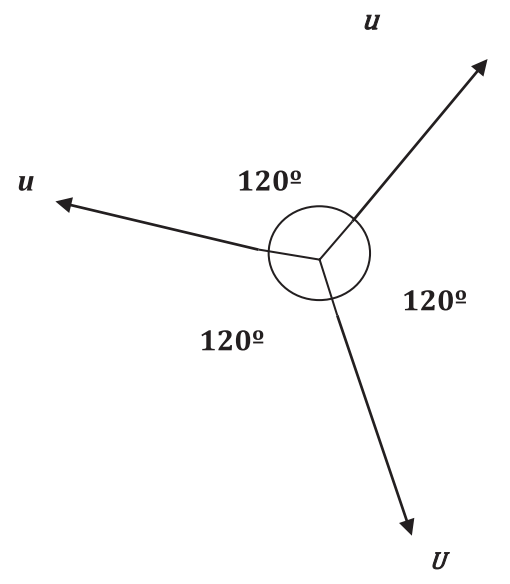

La suma de los tres vectores es nula, al igual que la suma de los tres colores da blanco, así como la suma de dos vectores es igual al tercero con el signo contrario, del mismo modo que la suma de dos colores es igual al anticolor del otro color. La analogía de la representación vectorial de las cargas con las propiedades cromáticas de los quarks en la cromodinámica cuántica es completa, constituyendo una descripción satisfactoria de sus propiedades físicas. $\mathrm{Si} \theta=120^{\circ}$, el producto escalar de un vector con cualquiera de los otros dos estará dado por:

$$
\boldsymbol{u} \cdot \boldsymbol{u}=e^{2 i \pi} \cos 120^{\circ}=-\frac{1}{2}
$$

Si a este valor le sumamos el producto escalar del primer vector con el vector restante tendremos:

$$
\boldsymbol{u} \cdot \boldsymbol{u}+\boldsymbol{u} \cdot \boldsymbol{u}=2 e^{2 i \pi}=-1
$$

igualdad que puede expresarse también como:

$$
\boldsymbol{u} \cdot(\boldsymbol{u}+\boldsymbol{u})=\boldsymbol{u} \cdot \boldsymbol{u}=e^{2 i \pi} \cos 180^{\circ}=-1
$$

es decir, las cargas se atraen. La proximidad de dos nucleones, protones o neutrones, en un átomo, produce una ligera asimetría en cada nucleón, dando lugar a un pequeño vector resultante que se complementa con un antivector de otro nucleón, o su respectivo anticolor, que es la fuerza residual fuerte que une a los protones a través de los neutrones, neutralizando su carga repulsiva eléctrica, como se ilustra en la figura.

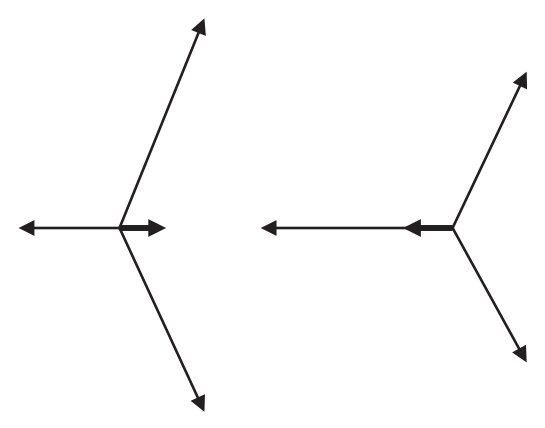


Esto da como resultado un empaquetamiento cúbico de los nucleones en un átomo, que es la que justifica la presencia de neutrones que hacen posible la unión de los protones, como se muestra por ejemplo en la figura que representa al átomo de berilio, el isótopo más estable de cuatro protones y cinco neutrones, en el que los protones están representados por las esferas rojas y los neutrones por las azules:

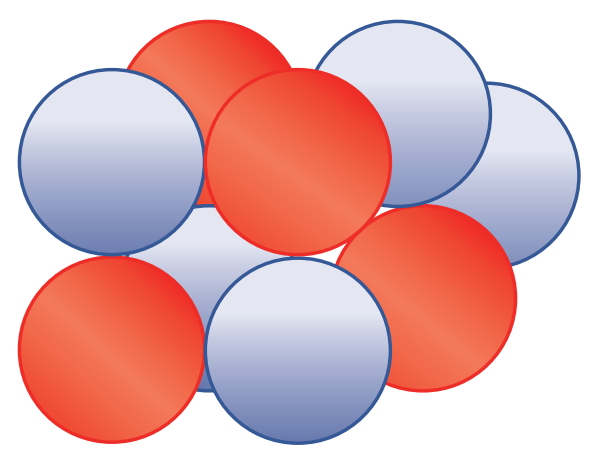

Resulta interesante comprobar que las cargas unitarias de las fuerzas fundamentales de la naturaleza pueden representarse en un campo vectorial complejo de cuatro dimensiones, como se ilustra en la figura, en el que las cargas de color de los quarks están representadas en el plano determinado por los vectores direccionales $i, j$, la carga electrostática por $k$ y la carga gravitatoria por li. La gravedad, la única fuerza que no ha sido unificada hasta el día de hoy, poseería una carga imaginaria, así como el campo gravitatorio y la masa gravitatoria $m i$, a diferencia de la masa inercial $m$ que sería de carácter real, concepto no contemplado por la física actual. En este caso, los vectores direccionales forman un ángulo $\theta$ de $90^{\circ}$, cuyo coseno es 0 , es decir, no hay atracción ni repulsión, consecuente con el hecho de que estas fuerzas no interactúan entre ellas.

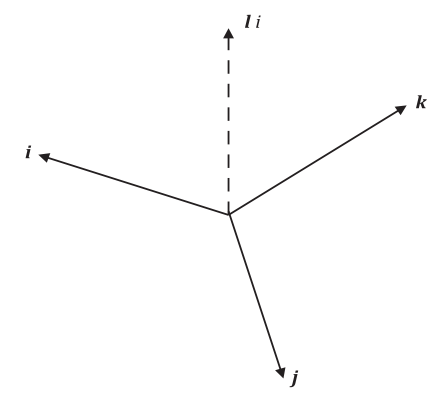

Por ejemplo, la fuerza electrogravitatoria entre dos electrones separados por una distancia $r$ estaría dada por el producto escalar de dos vectores complejos:

$$
F_{r}^{e^{-}}=\left(\frac{k e^{-}}{r^{2}} \boldsymbol{k}+\frac{G m_{e}}{r^{2}} \boldsymbol{l i}\right) \cdot\left(e^{-} \boldsymbol{k}+m_{e} \boldsymbol{l i}\right)=\frac{k e^{-2}}{r^{2}}-\frac{G m_{e}{ }^{2}}{r^{2}}
$$

\section{Universos de materia y antimateria}

Según el modelo expuesto, el vacío cuántico estaría conformado por diferentes subniveles en los que las partículas y antipartículas virtuales ejercerían una acción polarizadora sobre la carga de la partícula real, que puede ser un electrón o un positrón. Este proceso continuaría indefinidamente hasta el infinito. La energía total del sistema es nula. Puesto que el tiempo del positrón fluye del pasado hacia el futuro pero en sentido opuesto al del electrón, la suma total del tiempo es también nula, así como del espacio, conservando la simetría CPT. Esta propiedad se aplicaría a todas las partículas elementales de la naturaleza, es decir, a los leptones y quarks, con sus respectivas antipartículas. Podemos representar este proceso así:

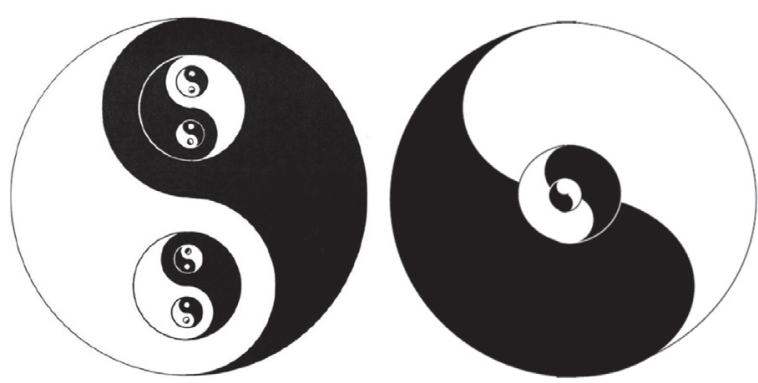

El diagrama de la izquierda representa el símbolo del yin y el yang reproducido al infinito de manera fractal, donde las áreas clara y oscura representan el protón y el positrón y sus respectivos subniveles cuánticos virtuales. En el de la derecha las dos áreas clara y oscura del círculo grande representan también el electrón y el positrón, en cuyo centro hay un círculo menor que representa el vacío cuántico con positrones y electrones virtuales, que interactúan con el electrón y el positrón polarizando sus respectivas cargas eléctri- 
cas. Estos círculos menores en ambos diagramas contienen a su vez otros círculos con positrones virtuales que interactúan con los positrones y electrones virtuales del vacío cuántico polarizando también sus respectivas cargas eléctricas. Este proceso continuaría indefinidamente hasta el infinito. El círculo menor del diagrama derecho que representa el vacío cuántico, simbolizaría la unión de los círculos menores del yin y el yang en el diagrama izquierdo. La energía total del sistema es nula. Puesto que el tiempo del positrón fluye del pasado hacia el futuro pero en sentido opuesto al del electrón, la suma total del tiempo es también nula, así como del espacio, conservando la simetría CPT. Esta propiedad se aplicaría a todas las partículas fundamentales de la naturaleza, tanto fermiones como bosones, es decir, a los leptones y quarks, con sus respectivas antipartículas, así como bosones y antibosones.

Ahora bien, si en el vacío cuántico hay tantas partículas como antipartículas virtuales, conservando el principio de simetría, es razonable concluir que podría existir un universo de antimateria que no observamos, pero que interaccionaría con el nuestro por el principio de incertidumbre de Heisenberg a través del vacío cuántico, que sería la frontera natural entre ambos universos. A escalas de Planck, los mantendría unidos, polarizando las fuerzas fundamentales de la naturaleza, incluida la gravedad. Si bien se observa únicamente la presencia de materia en el universo, los científicos no se explican a dónde fue parar la antimateria y el por qué de esta asimetría. Se ha especulado que en el big bang existía cien mil millones de antipartículas por cada cien mil millones más una de partículas, y que al enfriarse el universo se aniquilaron la materia y la antimateria, quedando un pequeño saldo de materia que es el que se observa en el universo. Esta hipótesis se basa en la suposición de que la violación de la simetría especular entre partículas y antipartículas en el mesón neutro $K$, se aplicaría también en la fase de unificación de las fuerzas en el big bang.
Proponemos que la violación de la simetría especular se daría también en el universo de antimateria, conservándose una simetría perfecta global entre ambos universos. En consecuencia, podríamos hablar de un universo de materia y antimateria, siendo su energía total nula, así como el espacio y el tiempo. Por consiguiente, el universo en su totalidad sería un vacío neutro, que denominamos neutrovacío como un concepto, resultado de la neutralización de las propiedades antagónicas del mundo fenoménico, sin espacio ni tiempo, ni materia, ni energía, cuya dinámica, según nuestra tesis, se manifestaría como materia, energía, espacio y tiempo, y a la vez antimateria, antienergía, antiespacio y antitiempo.

No es nuestra intención establecer una conexión entre el modelo propuesto y el taoísmo, apenas señalar cierta similitud, pues nuestra interpretación no corresponde a la concepción tradicional del taoísmo. Hawking fue muy despectivo al referirse a la obra del físico y budista Fritjof Capra, "El tao de la física", que establece analogías entre los descubrimientos de la física moderna y las enseñanzas del taoísmo, el budismo y el hinduismo, línea también seguida por el astrofísico vietnamita Trinh Xuan Thuan. En la filosofía occidental ocurre lo mismo. Hegel y Heidegger, por ejemplo, ignoraron por completo el aporte filosófico del Oriente, dentro de su visión eurocéntrica, que persiste hasta el día de hoy, no obstante la influencia del pensamiento oriental en filósofos occidentales, como el budismo en Schopenhauer, por ejemplo. Es innegable que el taoísmo y el budismo representan un intento de interpretación de la realidad, y que el Oriente ha priorizado esta búsqueda a través de un proceso de interiorización y meditación, a diferencia de Occidente que lo ha hecho a través de la razón y la exploración de la naturaleza. La afirmación de que la filosofía como tal surgió en Grecia y en consecuencia no pueden calificarse como filosofía las concepciones del Oriente, incluidas las del mundo andino, es una interpretación coherente 
desde una perspectiva occidental, pero que actualmente es objeto de debate.

Ahora bien, hemos visto que una partícula elemental real como el electrón, se polariza por acción de las partículas virtuales de diferentes niveles de energía del vacío cuántico. Estas partículas virtuales, a su vez, deben polarizarse del mismo modo que las partículas reales, a fin de no generar energías infinitas ni estallar bajo los efectos de su propia carga repulsiva. En consecuencia, cada partícula virtual de los diferentes subniveles del vacío cuántico sigue las mismas leyes de las partículas reales y están determinadas por las mismas ecuaciones. Teniendo en cuenta esta particularidad y siendo estos subniveles una secuencia infinita, cabría preguntarse si es posible que la partícula real sea a su vez una partícula virtual de un primer nivel de vacío cuántico de otra partícula real (de modo análogo al modelo del universo holográfico) que a su vez es una partícula virtual de otra real y así hasta el infinito, de manera que el diagrama esté incluido en otro mayor y así sucesivamente. Del modelo propuesto, se puede inferir la posible existencia de un universo de antimateria y de universos de materia y antimateria dentro de otros universos en una secuencia infinita de la cual el nuestro sería apenas un eslabón en la cadena de multiuniversos. Así por ejemplo, en el diagrama, nuestro universo estaría representado por una de las áreas, que contendría a su vez un microuniverso de materia y antimateria. Del mismo modo, nuestro universo con el universo de antimateria, estarían contenidos en un macrouniverso de materia y antimateria. Dentro de esta línea, el filósofo estadounidense Ken Wilber propuso que el vacío cuántico estaría conformado por un enorme amasijo de partículas virtuales enrolladas entre sí, formando una trama recurrente en el que la partícula real implica la existencia de muchas otras contenidas en la nube virtual que la rodea a medida que se desplaza, y cada una de las partículas virtuales arrastra su propia nube virtual, burbujas dentro de otras burbujas, y así sucesivamente al infinito, concepto muy similar al propuesto por nosotros, aunque sin la formulación matemática.

El problema del infinito ha sido abordado por diferentes pensadores a lo largo de la historia. El matemático Cantor hizo notables contribuciones al descubrir los números transfinitos que vienen a ser categorías diferentes de infinitos. Así, el número de infinitos decimales que contienen los números irracionales es mayor que el de los números racionales. Cuando hablamos de universos fractales contenidos unos dentro de otros en una secuencia infinita, conectados a través de subniveles de vacío cuántico, no estamos estableciendo necesariamente jerarquías de universos en cuanto a sus dimensiones, sino universos paralelos de materia y antimateria entrelazados por diferentes niveles de vacío cuántico. Una analogía nos puede servir de referencia. Si observamos el interior de un cilindro, veremos en perspectiva que los círculos son más pequeños a medida que se alejan de nosotros, pero en realidad son del mismo tamaño.

\section{Reflexiones filosóficas}

Una vez más, se pueden encontrar ciertas analogías con antiguas tradiciones orientales, en la cosmogonía tántrica por ejemplo, desde una cosmovisión teísta. Uno de los principios del taoísmo, establece que cada yin y yang contiene a su vez un yin y yang, que a su vez contienen otro yin y yang, y así sucesivamente. El movimiento raeliano, que se hizo conocido al afirmar que habían realizado la primera clonación humana, sostiene la existencia de universos dentro de otros en una secuencia infinita, desde una perspectiva atea. El símil del movimiento planetario y el modelo atómico de Bohr llevó a pensar a muchos que los átomos y moléculas era un microcosmos semejante al macrocosmos de planetas y galaxias, pero son realidades distintas gobernadas por leyes diferentes, la relatividad general y la mecánica cuántica. La teoría de los universos paralelos, realidades alternativas, los multiversos, ha sido planteada por la 
ciencia moderna y es uno de los argumentos de Hawking para refutar el principio antrópico fuerte y el diseño inteligente en su libro "El gran diseño", que trata de dar una explicación al ajuste fino de las constantes de la naturaleza que hacen posible la existencia de seres inteligentes como nosotros, sin recurrir a una intervención divina.

Se plantea ahora la siguiente pregunta: ¿Es el neutrovacío una realidad metafísica que trasciende el mundo fenoménico o una propiedad de la naturaleza misma que poseería una dualidad, el yin y el yang, el taita inti y la pachamama de la cosmogonía andina, para referirnos a algo cercano a nuestra realidad? La respuesta desde una perspectiva inmanente es que el neutrovacío es una propiedad intrínseca de la naturaleza, consecuencia de la coexistencia de universos de materia y antimateria. Desde el punto de vista kantiano sería el noúmeno, de carácter trascendente. Con relación a la perspectiva inmanente, podemos encontrar una analogía en las matemáticas. El número cero se obtiene a partir de la suma de dos cantidades iguales de signo contrario, una positiva y otra negativa. Matemáticamente, el cero existe como número e inclusive se realizan operaciones con él. No se trata, pues, de una cantidad que no existe y por tanto deba ser excluida del campo de las matemáticas, posee más bien ciertas propiedades que lo distinguen de los demás números, a diferencia, por ejemplo, del conjunto vacío.

Conviene hacer sin embargo una reflexión sobre este aspecto. Hemos señalado que en el modelo estándar de la física cuántica, así como en modelos alternativos como la teoría de supercuerdas, la teoría M y la mecánica cuántica de bucles, existe un límite en el tiempo y el espacio, que son el tiempo y la longitud de Planck respectivamente, más allá del cual no se puede definir la realidad. En nuestro modelo nosotros nos basamos en el concepto de límite matemático para la distancia cuando ésta tiende a cero, que no es lo mismo que el límite físico de la longitud de Planck. En el caso de una partícula elemental como el electrón, o cualquier otra partícula elemental, cuando el radio tiende a cero y en consecuencia el espacio y el tiempo, el efecto acumulativo de la antimateria y materia virtuales neutraliza la carga del electrón. En ese punto singular no existe el espacio, ni tiempo, ni materia, ni energía, no obstante se manifiesta en el mundo fenoménico con estas características que son las que observamos. Y esta particularidad se daría en todo el universo, que en esencia sería vacío, lo nouménico según Kant, manifestado en lo fenoménico, así como en la totalidad de todos los universos de materia y antimateria. La materia prima, en potencia, y la materia segunda, en acto, según Tomás de Aquino.

Entramos en el terreno filosófico. Lo nouménico y fenoménico coexistirían como aspectos de la misma realidad, como una sola entidad, no existirían el uno sin el otro, lo trascendente e inmanente se requerirían mutuamente. Siendo el neutrovacío la neutralización de los universos de materia y antimateria es, por consiguiente, la inexistencia de materia, energía, espacio y tiempo. Las ecuaciones aplicadas a las partículas elementales y la gravedad nos conducen a este resultado, al cual identificamos con lo nouménico, lo no manifestado. El tiempo del universo de materia transcurre igual que en el universo de antimateria, del pasado hacia el fututo, pero en sentido opuesto, de manera que se neutralizan. $\mathrm{Al}$ no existir el tiempo en lo no manifestado, es atemporal, eterno. Su manifestación es lo fenoménico, los universos de materia y antimateria, que a su vez son el sustento de lo nouménico. De manera que lo fenoménico y lo nouménico son aspectos de la misma realidad, no existe el uno sin el otro, son complementarios, se retroalimentan entre sí. Siendo así, el tiempo es eterno, no existe un comienzo ni un fin del universo, de lo fenoménico. Nos acercamos a la concepción budista de la realidad, el universo es infinito y eterno. 
Aquí entramos en un conflicto aparente con la cosmología vigente, según la cual el universo tuvo un origen en el big bang. Sin embargo, no todos los físicos están de acuerdo con este paradigma cosmológico. Roger Penrouse, notable físico y matemático británico de la Universidad de Oxford, defiende la idea de una cosmología cíclica conformal, según los datos recopilados por el satélite WMAP de la NASA, que presenta patrones circulares dentro del fondo de microondas cósmico, que sugieren que el espacio y el tiempo no empezaron a existir en el big bang, sino que nuestro universo se encuentra en un ciclo continuo a lo largo de una serie de eones, un ciclo de expansión y contracción de nuestro universo, que daría origen a un nuevo universo con nuevas leyes físicas, así como otro universo anterior al nuestro dio origen a nuestro universo, en una secuencia infinita sin comienzo ni fin.

Por otro lado, los astrofísicos Ahmed Farag Ali, de la Universidad de Benha, Egipto, y Saurya Das de la Universidad de Lethbridge, Canadá, afirman que la singularidad del big bang puede ser resuelta por su nuevo modelo, en el que el universo no tiene ni principio ni fin. Los investigadores usaron la ecuación de Raychaudhuri y las ecuaciones de Friedmann, que describen la expansión y evolución del universo, incluyendo el big bang, en el contexto de la relatividad general. El modelo de Ali y Das contiene elementos tanto de la teoría cuántica, como de la relatividad general. En términos físicos, su modelo describe el universo como lleno de un "fluido cuántico”. Los científicos proponen que este líquido podría estar compuesto por gravitones, hipotéticas partículas sin masa que median la fuerza de gravedad. Para entender el origen del universo, ellos analizaron el comportamiento de este fluido a través del tiempo. Sorprendentemente, encontraron que este no converge hacia la singularidad, sino que, al contrario, el universo parece haber existido siempre, si bien era más pequeño en el pasado.
Estos modelos son compatibles con nuestro modelo cosmológico en el sentido de que no establecen un origen del universo en el big bang ni un final. También es oportuno señalar que un grupo de estudiosos del concepto de "flecha del tiempo" liderados por el profesor Julian Barbour, del College Farm en el Reino Unido, ha intentado responder a algunos de los interrogantes que plantea el mismo. Los científicos sugieren que existen dos de estas flechas que se habrían formado durante el big bang, en el que habrían aparecido dos universos que se mueven igualmente a través del tiempo, pero en direcciones opuestas entre sí. Los investigadores aseguran que tras una serie de experimentos con un modelo sencillo de mil partículas su teoría reveló que moviéndose hacia atrás en el tiempo, hacia el desorden, uno finalmente saldría por el otro lado también en el orden después del big bang en un "universo espejo". Este "universo espejo" coincide precisamente con el universo de antimateria propuesto por nosotros, en el que el tiempo transcurre normalmente del pasado hacia el futuro, pero en sentido opuesto al tiempo del universo de materia. No obstante, según nuestro modelo, los universos de materia y antimateria existirían desde siempre más allá del big bang.

Los conceptos de cero e infinito están estrechamente vinculados con lo expresado anteriormente. En matemáticas, la teoría de límites permite trabajar consistentemente con estos conceptos que de otra manera darían lugar a inconsistencias para el desarrollo del cálculo. Newton y Leibnitz estaban conscientes de ello. Matemáticamente se expresa como $x \rightarrow 0$ y $x \rightarrow \infty$, es decir, $x>0$ y $x<\infty$, pero que tiende a cero e infinito respectivamente, que es el límite en nuestras ecuaciones para $r$. No obstante, cuando $r=0$, desaparece lo fenoménico, convirtiéndose, desde una perspectiva trascendente, en el noúmeno kantiano, más allá de lo cognoscible, a diferencia del idealismo de Fichte, Schelling y Hegel, que sostiene que el conocimiento de lo absoluto es accesible 
a la razón. Desde una perspectiva teológica, sería el lugar recóndito de Dios de las religiones monoteístas o el Parabrahman del hinduismo. Desde una perspectiva inmanente, como señalamos anteriormente, se trataría de una propiedad intrínseca de la naturaleza, despojada de lo trascendente, en cuyo caso la física iría más allá del límite de Planck. Independiente de ello, sostenemos que lo nouménico y lo fenoménico son inseparables y pertenecen a la misma realidad, y que las matemáticas nos conduce a ello, más allá del límite de Planck. Nuestra intención es mostrar una posible convergencia entre la filosofía y la ciencia.

El filósofo peruano Gustavo Flores Quelopana, miembro de la Sociedad Peruana de Filosofía, ha escrito un interesante libro basado en nuestro modelo, "La teoría cosmológica del Neutrovacio", desde una perspectiva teísta, en la que el neutrovacío juega el rol de la nada a partir de la cual Dios crea el universo, siguiendo una línea tomista, un creatio ex neutervacuum. El desarrollo de su pensamiento es elaborado y se encuentra en pleno proceso de investigación. Desde nuestra perspectiva agnóstica, como mencionamos anteriormente, sostenemos que lo nouménico y lo fenoménico son ontológicamente inseparables y pertenecen a la misma realidad, como las dos caras de una misma moneda.

Debemos reflexionar también sobre el hecho de que nosotros, nuestra propia consciencia, la vida, el nacimiento, la muerte, son una manifestación del mundo fenoménico, así como toda forma de materia, energía y tiempo. El llamado mundo espiritual, las experiencias religiosas y místicas, los fenómenos paranormales, los estados de consciencia en las prácticas de meditación, el despertar de los chacras en el yoga, serían todos manifestaciones del mundo fenoménico. En nuestro caso por ejemplo, hemos tenido experiencias místicas que podrían calificarse de extraordinarias, semejantes a las descritas por algunos mís- ticos, pero que sin embargo son independientes de nuestra posición filosófica. Según algunas escuelas budistas, estamos inmersos en la "rueda de la vida”, un mundo ilusorio que conlleva al sufrimiento, su objetivo es liberarnos de las ataduras del dualismo fenoménico extinguiendo el ego para alcanzar el nirvana, el shunyata o vacuidad, que es la ausencia de existencia real. Pero en el vacío absoluto no hay conciencia, no existe un observador ni nada que pueda ser observado, ni experimentado.

Cabe señalar que Hawking postula que la energía neta del universo es nula, puesto que la energía positiva de la materia y la energía negativa gravitatoria se cancelan entre sí, pero no se trataría de un vacío absoluto, que no estaría permitido por el principio de incertidumbre de Heisenberg, sino de un vacío cuántico con un estado de energía mínima, cuyas fluctuaciones, que consisten en partículas y campos que aparecen y desaparecen de la existencia, darían origen a la creación de una inmensa cantidad de universos posibles, unos $10^{500}$, sin recurrir a una intervención divina o a un diseño inteligente para explicar el ajuste fino de las constantes de la naturaleza, puesto que nuestro universo estaría dentro de un rango de probabilidades de universos con estas características. Según el modelo propuesto por nosotros, la teoría de Einstein se modificaría a escalas de Planck y la energía gravitatoria convergería a la energía intrínseca de la masa con signo negativo, como señalamos anteriormente, de manera que por el principio de simetría, existiría un universo de antimateria, cuya energía gravitatoria positiva convergería a la energía intrínseca de la masa con signo positivo, que se cancelaría con el de materia en un vacío absoluto. Lo mismo ocurriría con el vacío cuántico, que contendría un microuniverso de antimateria, el cual se manifestaría como un estado de energía mínima a través del principio de incertidumbre de Heisenberg, dando origen a una multiplicidad de universos, en un proceso de creación continua que contendría una infinidad 
de universos contenidos unos dentro de otros, como se ilustra en el diagrama anterior.

San Agustín plantea que con la creación se crea también el tiempo, siendo Dios un ser atemporal que se manifiesta en su propia creación. En el islamismo, la Tierra y los cielos eran una sola entidad conectada y homogénea, que posteriormente se formaron o separaron uno del otro. La cosmología moderna sólo se ha aproximado al tiempo de Planck, unos $10^{-43}$ segundos, más allá del cual no puede afirmar nada, apenas conjeturar, hecho que ha sido recibido con beneplácito por el Vaticano para sostener que más allá de ese límite está la intervención divina. En este contexto, cabe mencionar que con los experimentos del gran colisionador de hadrones, el CERN, un superacelerador de partículas, los científicos intentan recrear las condiciones iniciales del big bang y encontrar el bosón de Higgs -objetivo realizado recientemente- una hipotética partícula del modelo estándar de la física, más conocida como la partícula de Dios o partícula divina, que sería el origen de toda la masa del universo. Pero, ¿qué condiciones se dieron para que las fluctuaciones del vacío cuántico, como sostiene Hawking, dieran origen al universo y en qué momento se dieron? Para que estas condiciones se den tiene necesariamente que haber sido el resultado de un proceso, lo que conduce a una secuencia infinita de acontecimientos previos a la creación del universo y al inicio del big bang, lo que nos lleva a la conclusión de que existe un proceso de transformaciones que darían lugar a la creación de infinitos universos.

Otro aspecto a considerar es el tiempo cíclico, simbolizado en la antigüedad por el Uroburos, la serpiente que se muerde la cola, el eterno retorno. El tiempo lineal aparece con el cristianismo, con un comienzo y fin del universo, la caída del hombre y la salvación de Cristo, como un evento único e irrepetible. Existen teólogos que plantean la posibilidad, frente a los descubrimien- tos de la ciencia que nos muestran un universo de proporciones colosales, donde la Tierra no es más que una insignificante partícula de polvo perdida en la inmensidad del cosmos, que la caída del hombre debe interpretarse como un acontecimiento que se daría en todo el universo y en consecuencia la salvación también debería darse en todos los confines del universo. Hawking plantea la posible existencia de un tiempo imaginario en sentido matemático, circular, sin fronteras, sin comienzo ni fin, al igual que el espacio lo es en la relatividad general, considerando al universo como un tejido espaciotemporal curvo y cerrado, y un tiempo real en el que se daría el big bang, dando lugar a lo que él define como un universo autocontenido, que haría innecesaria la intervención divina. La idea de un tiempo circular no se contrapone con el modelo propuesto, si bien quedaría en el terreno especulativo. No obstante, la concepción de un tiempo infinito, plantea el problema de la imposibilidad de llegar al tiempo presente a través de un proceso de causa y efecto cuyo origen se encuentra en el pasado. Debemos entonces partir de una realidad concreta, donde el origen es el presente, e invertir el sentido del tiempo aplicando la causalidad hacia el pasado, en un proceso infinito, trátese de un tiempo lineal o circular. Siendo un proceso infinito, el presente es a su vez el pasado de un tiempo futuro infinito, en el que el pasado y el futuro convergen en un origen común que es la realidad ontológica del tiempo presente. Desde esta perspectiva, no habría un comienzo en el pasado ni un fin en el futuro, lo nouménico sería atemporal.

La posible existencia de universos de materia y antimateria nos conduce a la hipótesis de que se trataría de un mismo universo con dos manifestaciones que serían el reflejo de sí mismas, conservando una simetría complementaria. Siendo así, a cada partícula del universo le correspondería su respectiva antipartícula con propiedades opuestas, pero a su vez integradas en una misma 
partícula que las unificaría, en una interacción mutua, a través del vacío cuántico y el principio de incertidumbre, que nuestra consciencia percibiría como una misma realidad. Dos manifestaciones opuestas pero entrelazadas. A la pregunta ¿a dónde fue a parar la antimateria?, la respuesta sería "está en el universo mismo". Esta dualidad fenoménica estaría presente en todas las partículas elementales que conforman el universo, y en el corazón de cada partícula subyace la esencia nouménica del insondable neutrovacío. Asimismo, siguiendo esta línea de razonamiento, podemos conjeturar que los microuniversos y macrouniversos que se deducen del modelo propuesto serían réplicas del universo, una multiplicidad de universos integrados entre sí y percibidos como una misma realidad por nuestra consciencia.

\section{REFERENCIAS}

Alexandrov, A. D. Komologorov, A. N. Laurentiev, M. A. y otros. La matemática: su contenido, métodos y significado. t. I, II y III. Alianza Editorial, S.A. Madrid, 1981.

Álvarez Vita, Enrique. "La belleza como guía de la ciencia”. Revista Tradición, año XI, No 11, Universidad Ricardo Palma. Lima, 2011.

Álvarez Vita, Enrique. "Lo nouménico y fenoménico en un nuevo modelo del universo". Revista Archivos, año I, $\mathrm{N}^{\circ} 1$. Cenáculo de Filosofía Yachaywiñay. Lima, 2013.

Álvarez Vita, Enrique. "Universos de materia y antimateria”. Revista Tradición, año XIII, Nº 13, Universidad Ricardo Palma. Lima, 2013.

Álvarez Vita, Enrique. "Un esbozo sobre la unificación de las fuerzas fundamentales de la naturaleza y sus implicaciones filosóficas". Revista Evohé, año III, $\mathrm{N}^{\circ} 3$, Revista Villarrealina de Filosofia. Lima, 2014.
Alvizuri, Luis Enrique. Andinia, la resurgencia de las naciones andinas. IIPCIAL. Lima, 2002.

Capra, Fritjof. El Tao de la fisica. Editorial Sirio, S. A., Málaga, 1996.

Dalai Lama. El universo en un solo átomo. Editorial Grijalbo, S. A., Barcelona, 2006.

Davis, Paul. Superfuerza. Salvat Editores S. A., Barcelona, 1985.

Davis, Paul. La frontera del infinito. Salvat Editores, S.A., Barcelona, 1985.

Davis, Paul. El Universo accidental. Salvat Editores, S.A., Barcelona, 1986.

Einstein, Albert. El significado de la relatividad. Espasa -Calpe, Madrid, 2008.

Flores Quelopana, Gustavo. Eurocentrismo y filosofia prehispánica. IIPCIAL, Lima, 1998.

Flores Quelopana, Gustavo. Filosofia mitocrática y mitocratología. IIPCIAL, Lima, 2010.

Flores Quelopana, Gustavo. Mistica y neutrovacio. Revista Archivos, año II, $\mathrm{N}^{\circ} 2$, Cenáculo de filosofía Yachaywiñay. IIPCIAL, Lima, 2014.

Flores Quelopana, Gustavo. La teoría cosmológica del neutrovacio. Reflexiones sobre el modelo del universo del vacio cuántico fractal de Enrique Álvarez Vita. IIPCIAL, Lima, 2014.

Flores Quelopana, Gustavo. “La teoría cosmológica del neutrovacío”. Revista Tradición, año XIV, N${ }^{\circ} 14$. Universidad Ricardo Palma, Lima, 2014.

Feynman, Richard. QED: La teoría extraña de la luz y de la materia. Prensa de la Universidad de Princeton, Princeton, 1988. 
Haaser, N.; La Salle, J.; Sullivan, J. Análisis matemático. t I y II. Editorial Trillas S. A., México, 1972.

Hawking, Stephen. Historia del tiempo. Editorial Grijalbo, S. A., Bogotá, 1989.

Hawking, Stephen. La teoría del todo. Debate. Barcelona, 2007.

Hawking, Stephen; Mlodinow, Leonard. El gran diseño. Editorial Crítica, S. L., Barcelona, 2010.

Hawking, Stephen; Penrose, Roger. La naturaleza del espacio y el tiempo. Random House Mondadori, S.A., Barcelona, 2011.

Miró Quesada, Francisco. Las supercuerdas. Empresa Editora El Comercio S. A., Lima, 1992.

Olivencia Baldasari, Luis. "El neutrovacío como elemento de la continuidad científica”. Revista Archivos, año II, $N^{\circ}$ 2, Cenáculo de filosofía Yachaywiñay. IIPCIAL, Lima, 2014.

Penrose, Roger. El camino a la realidad. Quebecor World S. A., México, 2008.

Resnick, R., Holliday, D. Física. t 1 y 2. Compañía Editorial Continental, S. A., México, 1979.

Santaló, L. A. Vectores y tensores. Editorial Universitaria de Buenos Aires, Buenos Aires, 1964.

Sears, F., Zemansky, M. Fisica. Aguilar S. A. Ediciones, Madrid, 1972.

Spiegel, M. R. Análisis vectorial. Serie Schaum, McGraw-Hill, México, 1979.

Wilber, Ken. Sexo, sexología, espiritualidad: el alma de la evolución. Gaia Ediciones, Madrid, 2003. 